\title{
VICTOIRES ET COLONNES DE L'ALTEL FÉDÉRAL DES TROIS GAULES : DONNÉES NOUTELLES
}

\author{
Par A. Acon et P. QroNiam
}

La découverte, an février 1958, des deux tiers de l'inscription dédicatoire de l'amphithêatre de la Croix-Rousse ${ }^{1}$. l'exploration des vestiges de cet édifice, menée à grands frais. depuis cinq ans, par la Ville de Lỵon avec le concours de l'État ${ }^{2}$, les études que ne cessent de susciter cette trouvaille et ces recherches. comme en témoigne par ailleurs le présent fascicule de Ciallia ${ }^{3}$. attirent à nouveau l'attention sur le sanctuaire qu'en 1?2 arant notre ire les soixante peuples des Trois Gaules dédièrent aux divinités protectrices de L'Empire. Rome et Auguste. La localisation et la disposition générale des principaux éléments de ce lieu de culte ne soulevent plus aujourd'hui de graves controverses ${ }^{4}$ : sur la pente méridionale de la colline (fig. 1), à cheval "sur l'arête faitière qui partage la déclivité de la còte ("n deux versants opposés "5, une vaste terrasse, longue de $296 \mathrm{~m}$. d'Est en Ouest el large de 69 du Nord au Sud, fut aménagée et mise en communication aver les quartiers bas it la ville de Lugdunum par une double rampe monumentale, au sommet de laquelle se dressaient sans doute l'autel fédéral et les deux colonnes qui encadraient ce dernier. Du ròté de l'Ouest, la terrasse s'étendait jusqu'à l'amphithéâtre ; à l'Est, jusqu'à la falaise: qui dominait un bras du Rhône. C'est aux abords de son extrémité orientale qu'a été trouvée la pièce que nous présentons icio.

Elle a élé mise au jour. le 13 janvier 1961, au cours de travaux d'édilité elfeclués au carrefour de la rue des Fantasques et de la rue Grognard. sur le revers oriental de la Croix-Rousse, au-dessus du débouché du tunnel roulier. I"n effondrement du sol à cet endroil, il $y$ a une dizaine d'années. et, par la suite. de nouveaux tassements décidèrent, en juillet 19:59, les services municipaux à entreprendre de profonds sondages. d'autant plus nécessaires que la zone menacée par ces mouvements est bordée, du còté du fleuve, par un escarpement de quelque quarante mitres de hauleur. Asse\% rapidement fut dégagée l'ouverture d'un puits carré, de $1 \mathrm{~m}$. 90 de còté, entièrement maçonné et

(1) J. Gurret A. Acdx, La dedicare de l'amphithéalre des Trois Gaules, dans le Bull. des musées lyonnais, 1958, p. 151 (59)-159 (67); J. GuW, dans les Comples rendus de l'tcad. des Inscr. el Belles Lellres, mars 1958.

(2) G. P. Qonian, Gallia, XIX (1961), Informations archéol., p. 4.40-441.

(3) P. 117 et suiv.

(4) Cr., en dernier lieu, les descriptions de A. Aros, Essai sur la topographie de I.ugdunum, p. 14\%-160, et A. Grixise, Manuel d'archéologie gallo-romaine, IV, 2, p. 506-5l6.

(5) Ph. F.ris, La table claudienne de L.yon, p. 12.

(6) Cous adressons nos remerciements au directeur des services techniques de la Ville de Lyon, II. Gsell, ainsi yu’a .1. Bertharion, directeur du service de la Voirie, et à ses collaborateurs, III. Septier, (iaudet et Poncet, qui signalirent la découverte de cette piece et, de differentes manieres, voulurent bien en faciliter la publicalion. 


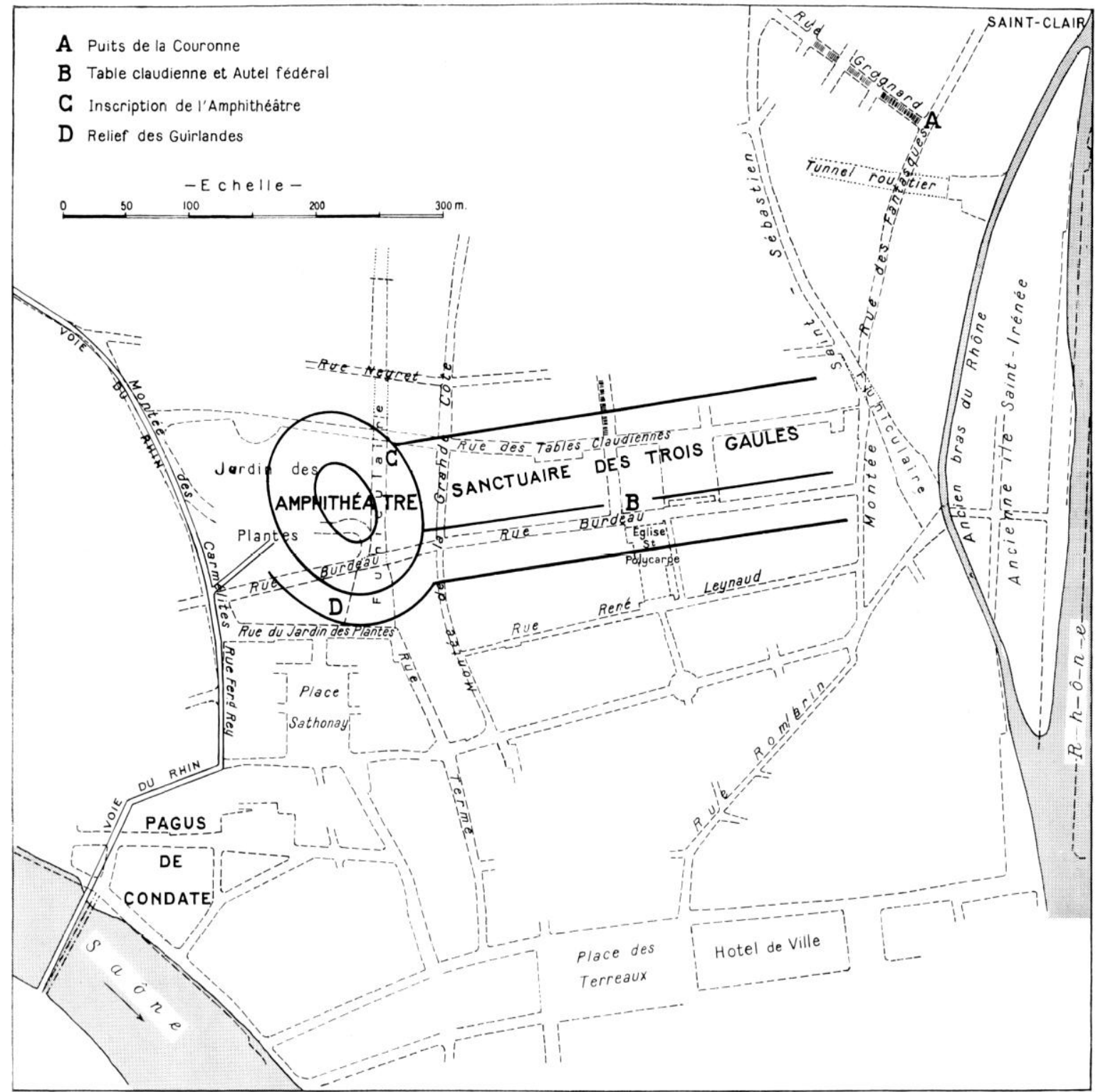

Fig. 1. - - Plan du quartier du sanctuaire fédéral des Trois Gaules, a l.yon. Sont indiques par des lettres les points ou ont ete decouverts la couronne dite des Fantasques (A), la Table Claudienne (B), l'inscription de l'amphithéatre (C) et les framents du relief des Cinirlandes $(\mathrm{D})$.

plongeant d'un seul trait de la cote :20:3 is la cole 178 . oi il rencent rait une premiere walerie. pour

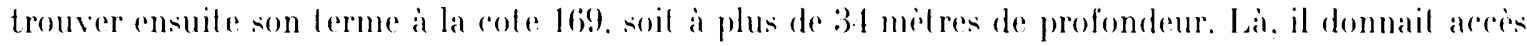
a un systime de galeries: une dizaine sensiblement Nord-sud. creusés à des niveaux progressivement plus bas a mesure que l'on approche du fleuve. la plus orientale se l rouviant mème au-dessous du niveau du lit, du Rhòne (cote 162 au pont Morand) . . et unies ent re elles par ciny autres. perpendiculaires; en trois points. correspondant i des croisements. al parurent d'aul res puits. semblables au premier mais non encore déblavés. Laroes de $1 \mathrm{~m}$. 30 à $1 \mathrm{~m}$. 90, hautes de $1 \mathrm{~m}$. 80 ) à $3 \mathrm{~m}$. 10. (es galeries él aient, elles aussi, maconnées el possédaient le plus souvent un radier en dur. On les retrouva loutefois mal conservées; et le sable naturel qui s'écoulail de leurs fissures ralentil sérieusement. la marche des travaux. déja gènés par le remblai précipité lans les puits el qui tendail à envahir les galeries voisines. Lu surplus. il parut indispensable de procéder en certains points à des 


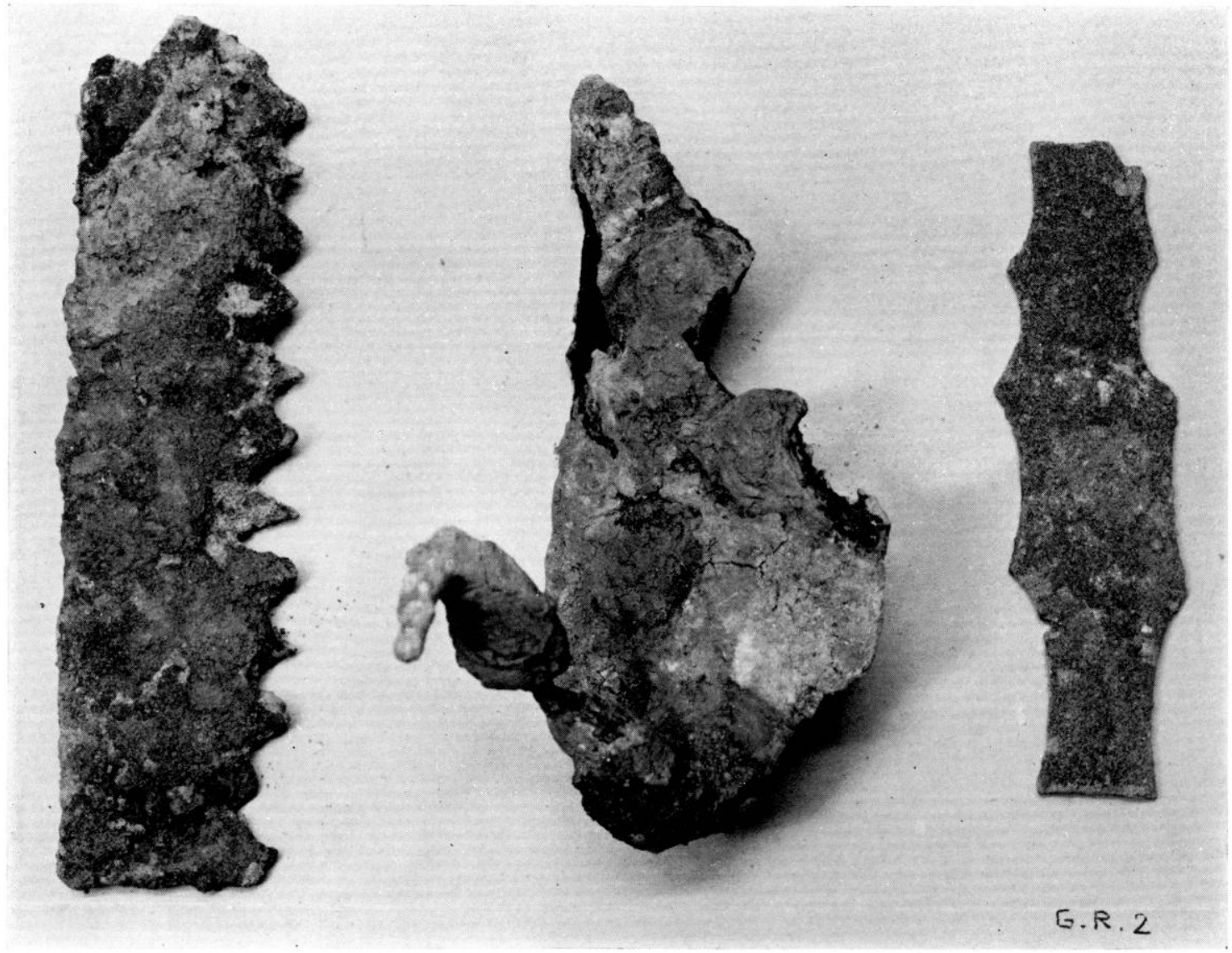

Fig. 2. - Scie, truelle et lame de bronze antiques retrouvées dans les galeries des Fantasques, à Lyon.

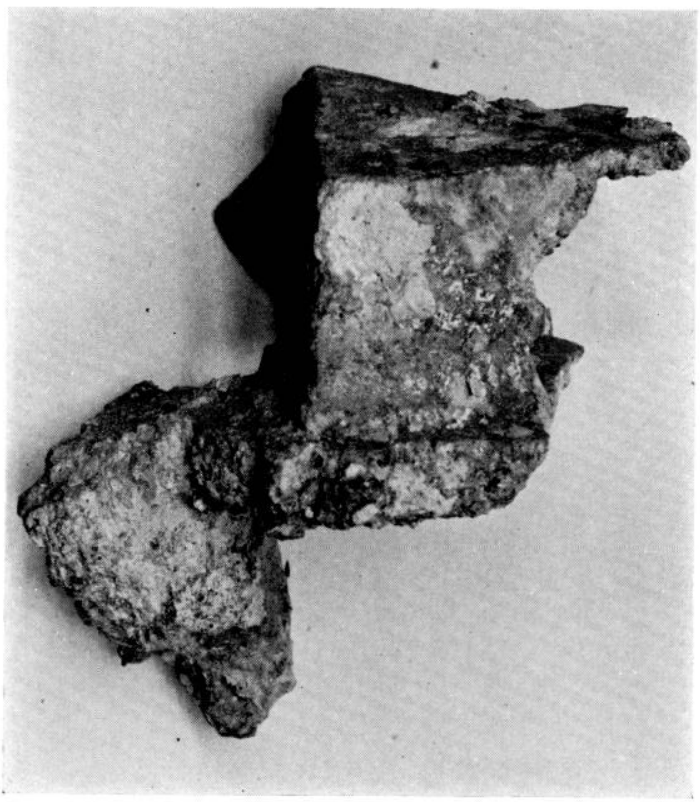

Fig. 3. - Angle de socle en bronze, ibid. 
ronsolidations, qui ne purent ètre entreprises que par l'ouverture d'une galerie d'approche partant. de la cour d'un immeuble voisin du tunnel et pénétrant de plain-pied dans un des éléments inférieurs du systeme.

sur la date de construction de ce réseau et sur sa fonction. les recherches d'archives n'ont pas fourni le moindre renseignement ; tout au plus savons-nous, par st eyert ? que l'architecte Chenavard le visila, partiellement, vers $1860^{8}$. On peut seulement affirmer, apris examen des maconneries. qu'il ne s'agit pas d'un ouvrage antique. A peine mieux renseignés en ce qui concerne son remplissage. nous observons cependant que le remblai de ses parties dégagées les plus basses comporlail mêlés a des matériaux et à des déchets modernes, de nombreux resles d'époque gallo-romaine : des: fragments de poteries el d'objets métalliques que les services techniqques de la Ville remirent, aver un louable empressement, au musée archéologique. La présence et, par la mème occasion. l'origine de ces vestiges n'offrent pas, a notre avis, de myst ire si l'on se rappelle les grands brassages de terres et déplacements de déblais qu'entrainèrent les l ravaux entrepris au xide siecle sur le sile. voisin. du sancluaire fédéral : agrandissement du chrrur de l'église saint-Polycarpe. abaissement de la rue Burdeau, ouverture de la tranchée du funiculaire. opérations qu'il faul cerlainement metlre en relation arec le comblement du "systeme des fanlasques".

Parmi ces restes antiques (fig. 2 et 3 ) figure la pièce qui fait l'objet de cette étude : la moitié d'une grande couronne de laurier en bronze (fig. 4 et 5). I)'un poids de $7 \mathrm{~kg}$. 50), et d'un diamètre de $0 \mathrm{~m}$. $46^{9}$, elle présente à une extrémité une tige longue de $10 \mathrm{~cm}$. of épaisse de 2 à 2 (m. ந. Au-delà s'amorcent dix rangées de feuilles lancéolées, longues de 10 à $11 \mathrm{~cm}$., large de $3 \mathrm{~cm}$. 5 et se recourrant de $4 \mathrm{~cm}$. Chaque étage ne comporte que trois fevilles, une à l'extérieur de la courbe, deux sur les côtés; celle qu'on attendrait à l'intérieur fait défaut, sans doute parce que, sous l'angle où était vue cette couronne, elle ent. été dissimulée par less feuilles latérales ${ }^{10}$. La quatrième et la septième feuilles d'un dess ròtés sont munies de pattes, larges de 3 ( $\mathrm{cm}$.. en saillie de $1 \mathrm{~cm}$. jo à l'intérieur de la courbe. L'une de ces pattes montre encore en place le rivet qui la traversait et dont la tête mesure 1 'm. 5) de diamètre : il maintenait une pièce relativement mince, aujourd'hui disparue et dont nous reparlerons. La patine, de ce beau vert bleu qui caractérise le bronze dit. rorinthien, est rehaussée par les larges plages encore recouvertes de la feuille d'or qui habillait toute la pièce, à l'exception de la tige terminale et de la partie inférieure qui étaicnt invisibles.

Un examen attentif de cette couronne, du moins de l'important fragment qui en est conservé permet de relever quelques données quant à sa position dans un ensemble monumental. Il est exclu qu'elle ait été placée contre une paroi, muraille ou autel. car clle aurait présenté. à cette paroi. une face lisse et non décorée. Or, lisse et non décorée. tefle est. justement la tige terminale qui, sans aucun doute, devait s'engager à l'intérieur d'un support tres vraisemblablement la main d'une statue; et les proportions mèmes de la couronne impliquent une statue au moins double de la grandeur naturelle. Linfin, le fait. que l'intérieur de la couronne n'offre pas de rangée de feuilles donne a prenser que l'objet était vu a distance et par dessous, qu'il était donc présenté à une assez grande hauteur.

(7) Nonvelle histoire ale L,yon, I, p. 125-126, fig. 190.

(x) Il y reconnut, à tort, un sout romain.

(9) longueur de la piece developpie : 0 m. 65 .

(10) Plusieurs feuilles et la tione terminale sont tordues; elles l'ont éte accidentellement, sans doute lors du bris de la couronine. 

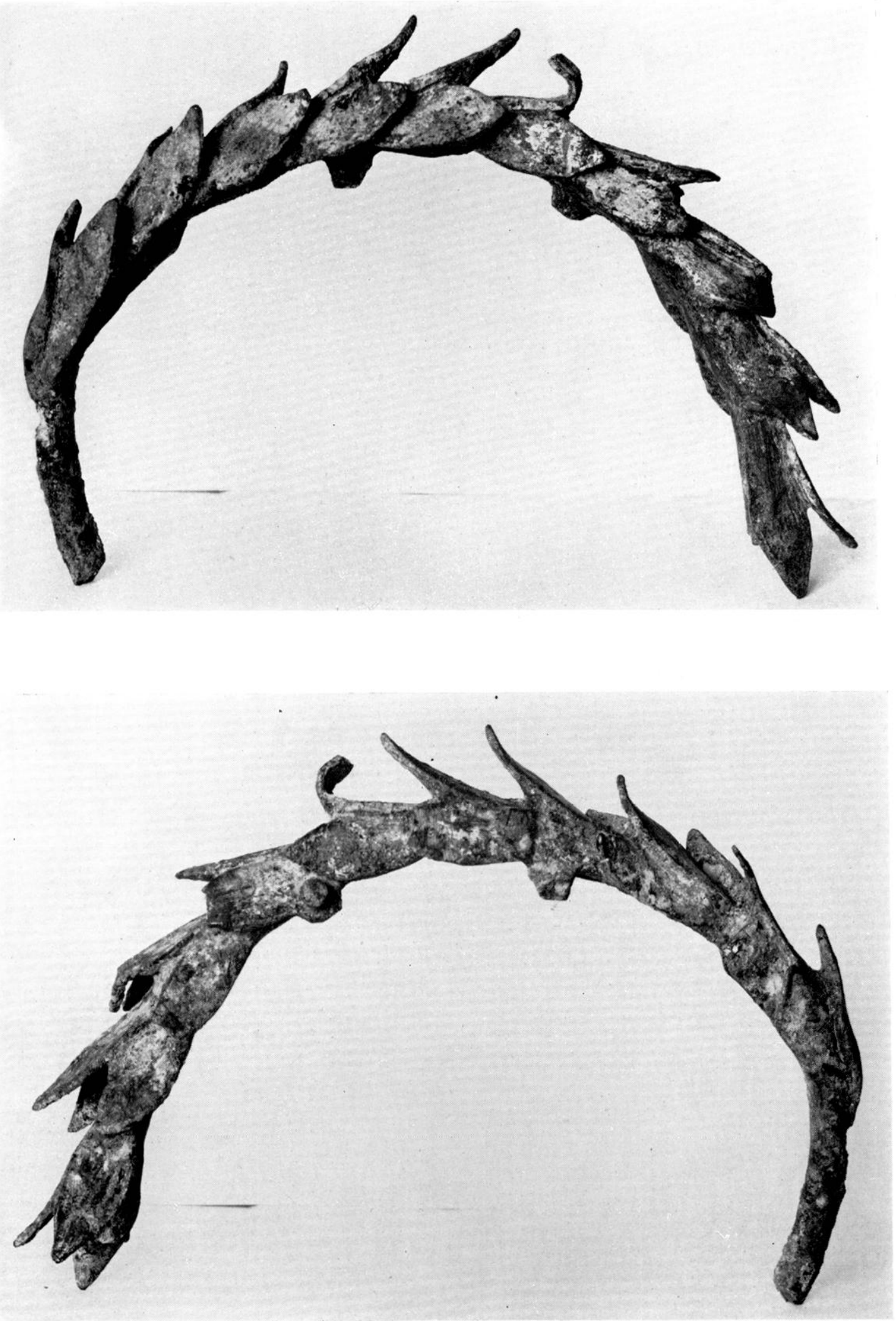

Fig. t et 5. ... Ioitie de couromne de laurier en bronze, ibill. Face et revers. 
Provenant done, selon toute probabilité, de la zone du sanctuaire et tenue, croyonsnous, par un personnage deux fois plus grand que nature. cette couronne de laurier peut. sans excès d'optimisme. être rapprochée de celles que brandissaient les Victoires dressées de part el d'autre de l'autel fédéral des Trois Ciaules. Pas de monument antique aussi typiquement lyonnais que cot autel encadré de deux colonnes portant des personnages dont la silhouette mème suggère qu'ils étaient de bronze : impossible, en eflet, de lailler dans le marbre, surtout pour les exposer à une telle hauleur. des éléments aussi découpés que palme et couronne.

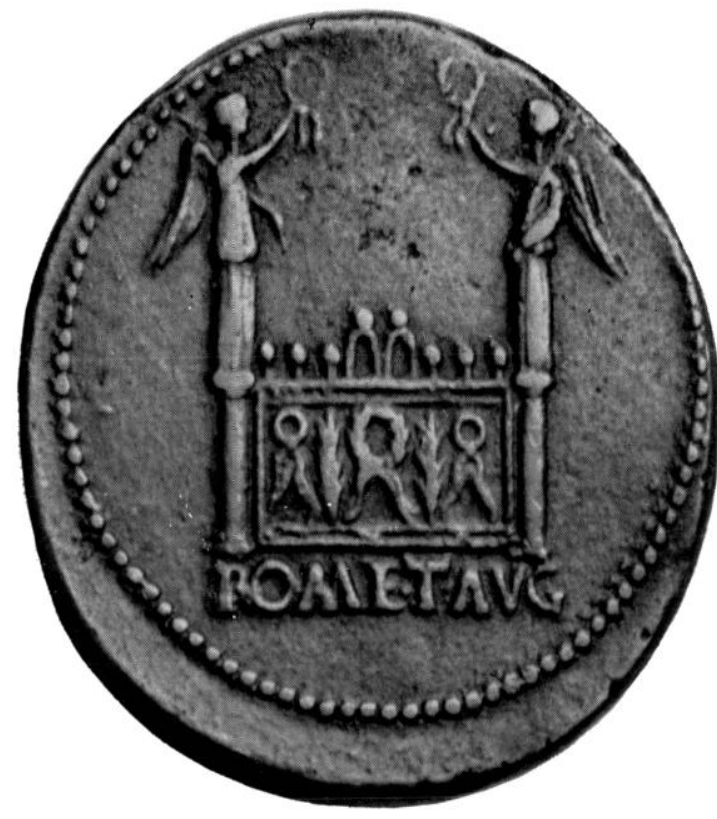

Fin. li. Bromze de Tibre ant revers de lanlel

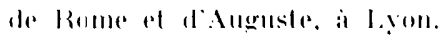

Combreux sont, on le sait, les hronzes impériaux figurant au revers cet autel de Rome at d'Auguste. Frappés à Lugdunum à partir de l'an 1: avant .J.-li.. pour l'onsembla de la ciaule. ils se répartissent entre vingetquatere types. compte non temu des copies des empereurs gaulois du $11 \mathrm{e}$ siècle : six avere l'effigie d'Auguste, quinze avee celle de Tibere. deux pour Claude et un pour Néron ${ }^{11}$.

$\therefore$ ilimatge de l'autel apparail passablement allére, interprété sur les coins de Claude of de

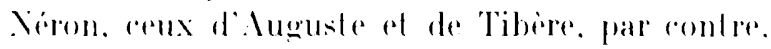
figurent le monument selon un ranon assez constanl "l. sirement. de lacon plus fidite ffig. 6: : sur un sorle qui porte invariablement la légende $R(0) .1$ ET . MCi. yu'il faut considerer comme un simple exergur. sérige lautel. plus large que hand, la latere principale ornere d'une couronne acestese de deux branderes de laurier. el. aux extrémités. de deux trépieds. surmontes chacun d'une couronne. Cout-

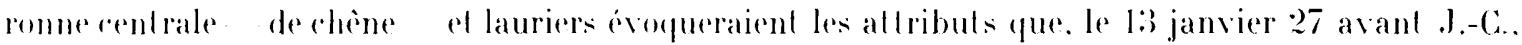
le sénal décerna, avec le nom d'Auguste, a oclave pour rélébrer sal victoire et le remercier d'avoir saure les ciloyens ; les trépieds porteraient les couronnes de laurier - consacrant le titre de Grand Pontife. qui lui fut attribué quinze ans plus tard. sur l'autel sont disposés d'autres ornements. variables d'un type monélaire i l'autre. le plus souvent des trépieds au nombre de six ou de huil. sensiblement plus hauts. les deux du centre portent des couronnes ef des orbes. symboles de la puissance souveraine el de l'efficacile de Rome el d'luguste sur l'univers entier: les trépieds

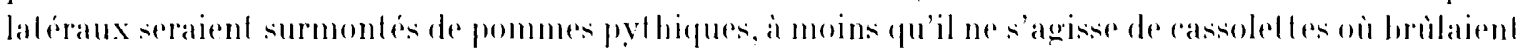
lencens. Les uns of les aul ress sonl parfois concadres par des hastes dressées ${ }^{12}$.

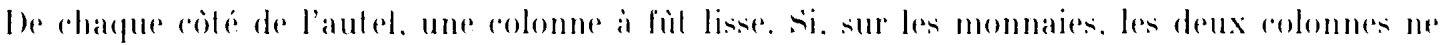

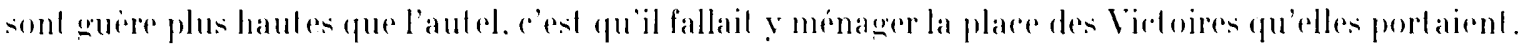
se laisant fare. les Victoires tendent le bras droil. la main sensiblement plus haule que l'épaule.

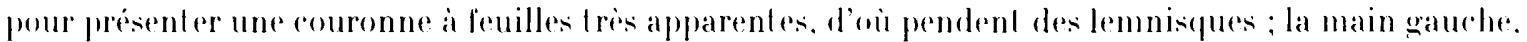
ramenéce le long du corps. lienl une palme appuyée contre l'épaule. L'allitude est calme'; les vite-

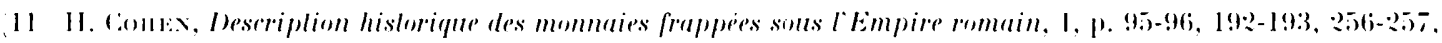

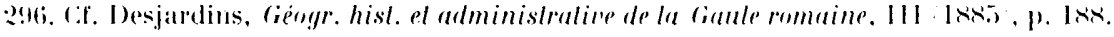

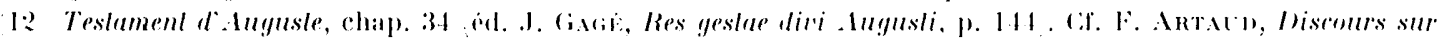

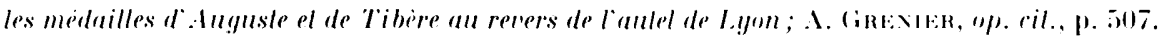




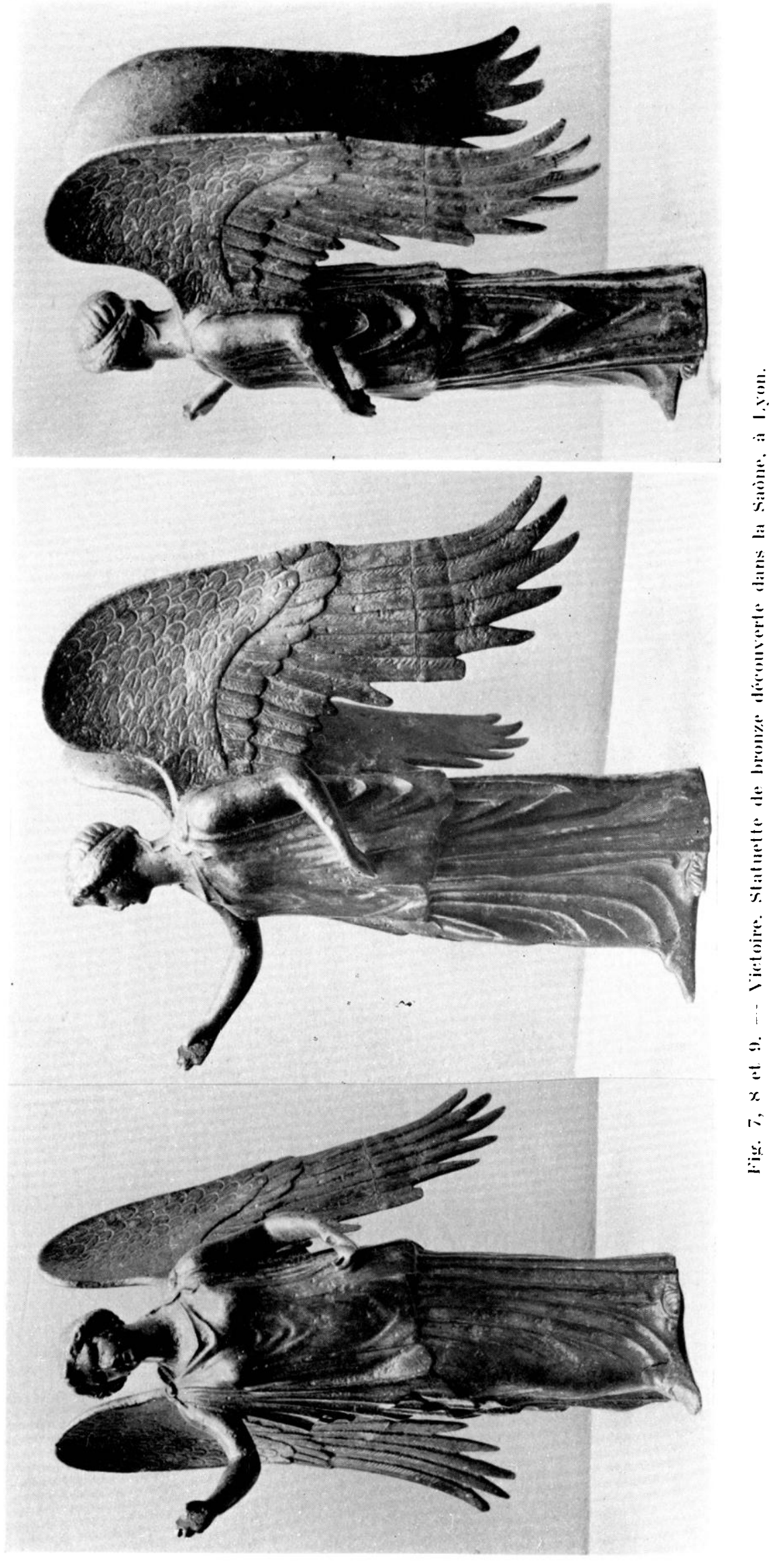


ments dessinent de longrs plis verticaux : ce n'est qu'au lemps de Claude que res personnes s'animent d'un mouvement qui. gonflant leur robe, semble les port er l'une vers l'aut re; ; simult anément. des cannelures apparaissent sur les colonnes.

In autre document. au reste, nous oflere de l'avis grénéral. une image plus précise de ces Virtoires, telles que les monnaies d'Auguste et de Tibere en esquissent la silhourthe, calme et majestueuse : une (harmante statuetle de bronze. haute de 0 m. 28 et lourde de $3 \mathrm{~kg}$. 980 , trourée en 1866 dans le lit de la Saòne. juis de l'actuel pont Kitchner (fig. 7. 8 ot 9$)^{13}$.

Classique est. ici, l'opposition de la ligne des épaules et de celle des hanches : si le corps repose sur la jambe gauche. toute la partie droite est portée en avant. le bras pour tendre la couronne, la jambe pour accompagner le geste ; la tite elle-meme, petite, haut perchée et inclinée sur la droilte. suit le mouvement que trahil, enfin, le drapé des vìtements. Encadrant le visage de deux bandeaux, la chevelure, sans chignon. est relenue par deux rubans; noués sur la nuque. ils relombent sur les. larges épaules. derrière lesquelles s'altachent de grandes ailes aux belles rémiges ${ }^{14}$. Ouvert en triangle sur la poitrine, plaqué sur les seins et le ventre. le chiton, passé sous le bras gauche et retenu aux épaules par des fibules. relombe sur le còté droit en grands plis rectangulaires, les extrémilés de cette pirce d'éloffe descendant ainsi plus bas que le grenou. Symétriquement, la longue robe, qui ne laisse voir que la pointe des pieds. forme sur le colté gauche une autre masse de plis qui équilibrent reux du chilon. Mais ce qui, dans cetle statuetle. appelle surloul not re altention. c'est le geste de la main droite. porlèe en avant au niveau de l'épaule : elle ne se cambre pas pour lenir la couronne. Xous en déduisors que celle-ci n'étail pas saisie par le bas. mais par le colé ; en d'autres lermes. pour prendre une image commode : non par le point de th heures du cadran. mais par celui de t.

Cette dernière remarque n'est pas. croyons-nous, sans intérèt du point de vue de lïdentifieation et, en mème temps. de l'interprétation de la couronne des Fantasques. On se souvient des deux pattes rentées sur les fenilles des quatrième et septième rangées, auxquelles étaient fixées des acerssoires dont il ne reste rien : la tige terminale étant engagée dans la main porteuse et la partie feuillue constituant la moitié inférieure de la couronne. res pattes ne seraient-elles pas des points d'attache de lamelles de métal par ailleurs reliées a la main ? I)e celle-ci. en effet, la seule cmprise, néerssairement imparfaite, ne pouvait suffire à maintenir en place. immobile, un objet d'un tel poids et d'un tel développement. entièrement exposé, en outre, à la pression parfois violente des vents qui balaient la colline. Et l'on peut supposer que, bien calculée, la disposition des lemnisques, qui s'échappaient. de la main, oftrait le moyen, à distance. de dissimuler, au moins partiellement, cess lamelles.

Il aurait. été étomnant. enfin. que les Victoires de l'autel fédéral n’apparussent pas dans l'imagerie locale fournie par les médaillons d'applique de la vallée du Rhòne. Trois l'entre rux les evoquent effertivement, mais sans en donner une représentation fidèle:

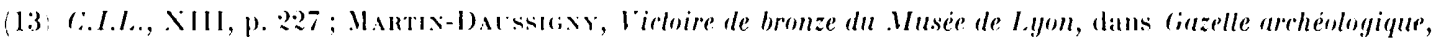

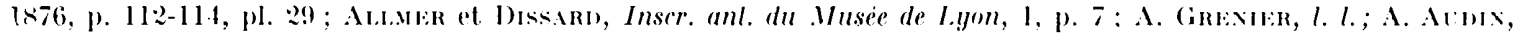
"p. cil., p. 153. Lille vient de faire tout recemment l'ohjet d'une restauration, confice au centre de recherches de l'Histoire de la Siderurgie, a Nancy : l’aile droite, hrisée et tordue, a ite redressie. Il reste à déplorer, sans doute, la perte de la palme et de la couronne, et des doigts qui les tenaient : mais lattitude du persommage fixe les idees quant aux gestes des mains mutilies.

(1) Par une amusante maladresse, ses ailes, qui a löchelle humaine mesuremient 1 m. 30 de hant environ, sont fixies aux omoplates par-dessus lo vètement. 
ils les mettent en scène dans des ensembles où la part active qu'elles prennent leur impose des attitudes différentes de celles du modile.

Le premier (fig. 10) est celui où le céramiste Imator soroupe une série de personnages suggérant symboliquement Lugdununi ${ }^{15}$. tu sommet. au-dessus d'un dieu dont l'identité incerlaine est ici

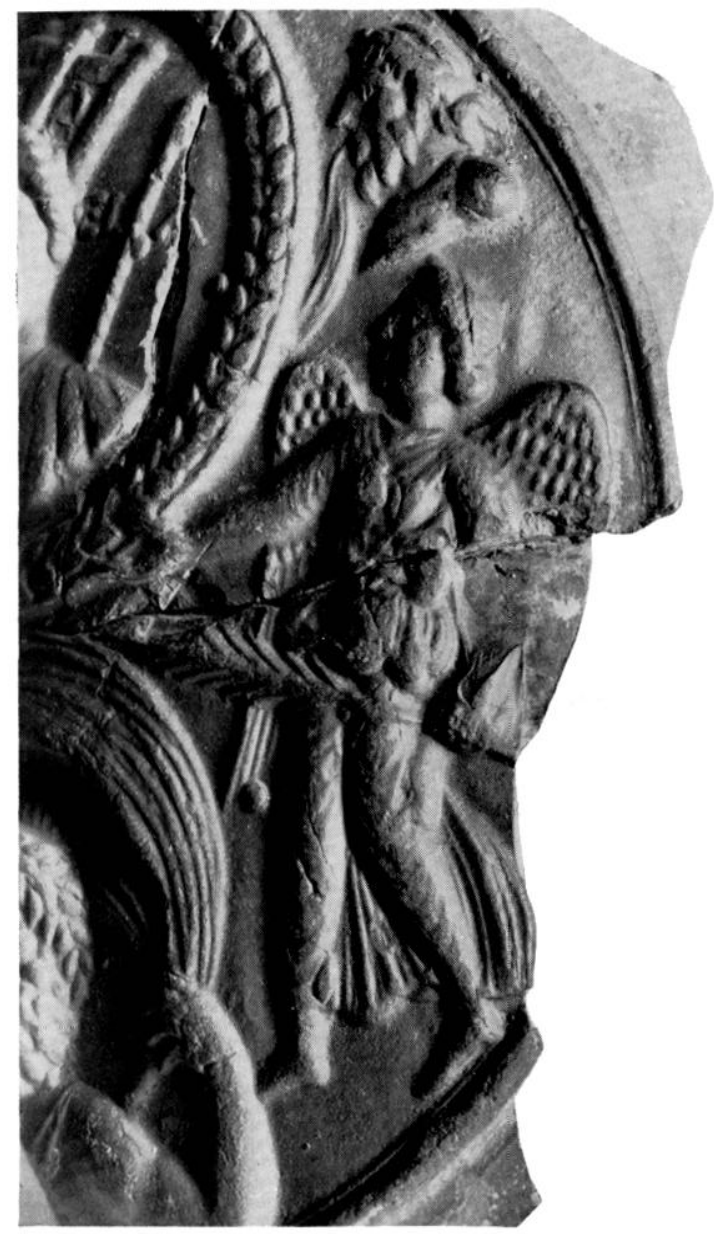

Figr. 10. - Me Maillon d'Amator. Fragment trouve à Viemne: Victoire. sans intérèt, parait la Tutelle de la ville dans une couronne de laurier: de part el d'autre. deux bustes barbus crachant de l'eau figurent les deux lleuves lyonnais; dans les parties latérales du médaillon, deux Victoires : seule, celle de droite subsiste. L'ensemble ayant pour objel l'exallation de Lugdunum, il est clair que ces Victoires sont. relles de l'autel fédéral. Cependant. pour répondre

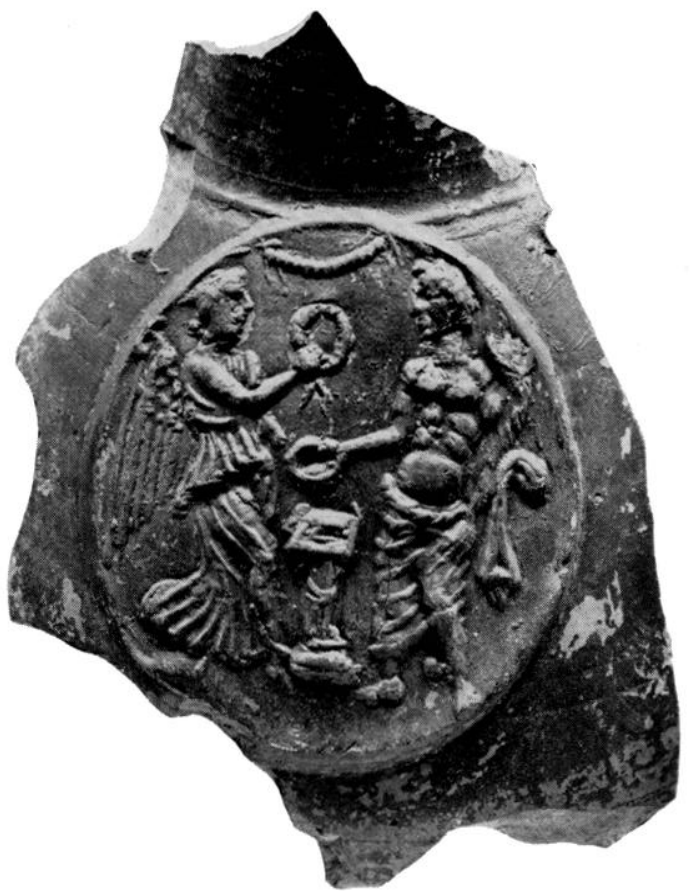

Fig. 11. -.. - Jimgment de médaillon d'applipue trouve à Vienne: Victoire et Gínie de Lugdumum.

aux exigences de la "mise en cercle", leur allitude a élé modifiée : la seule qui soit conservée lient. de la main droite une palme placée horizontalement; du bras droit. abaissé. elle soutient bien une couronne de laurier. mais grossie démesurément pour servir de cadre au buste de Tıtela. A còlé de ces déformations systématiques. il en est d'involonlaires : la robe, par exemple, n'est plus la tunique longue et droite, mais la stola, ceinturée sous la taille et retombant en un large repli. C'est dire qu'Amalor n'apporte pas grande précision quant a l'apparence de la Victoire fédérale; il l'a représentée sans se préoccuper des détails.

Le second médaillon (fig. 11), tardif, associe une Victoire au Génie de Lugdunum ${ }^{16}$, debout,

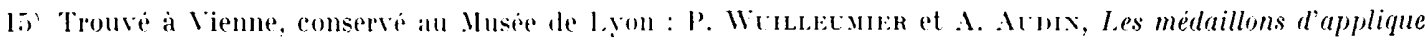

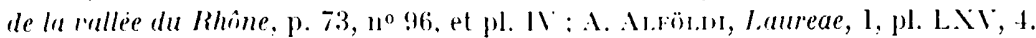

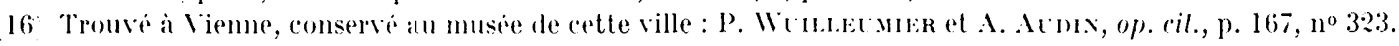




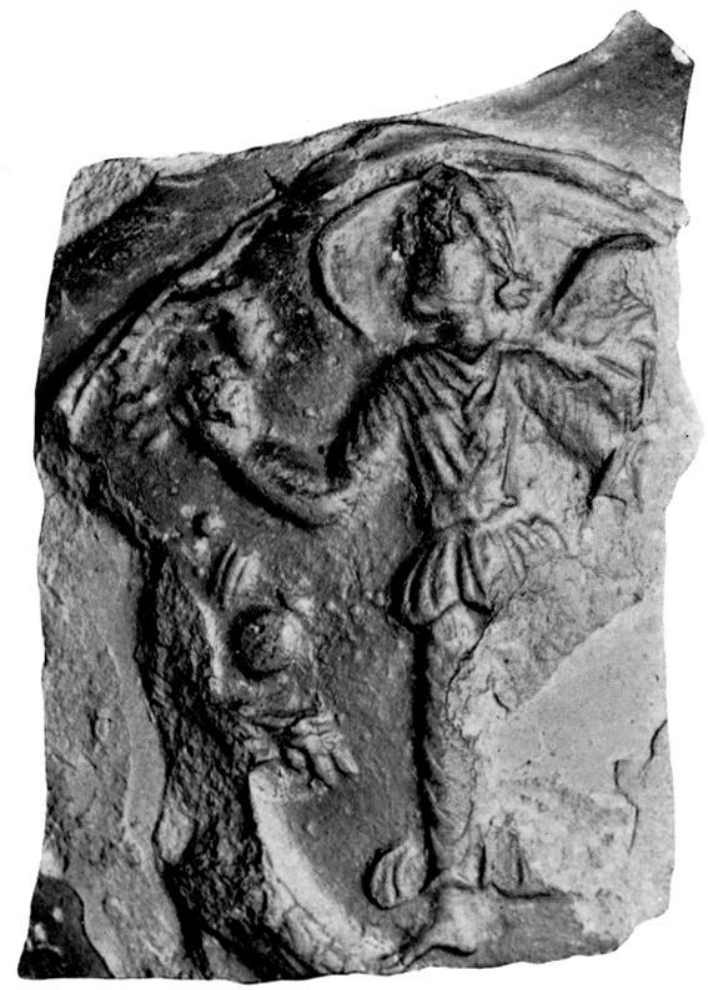

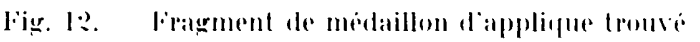
at Saintertolombe : Victoire.

lourné ì gauche. lendant la main droite vers un aulel ou il fail une libation : la gauche tient la corne d'abondancer qui est son all ribul signalétique. lin face de lui, a gauche. la Vicloire. dans un vitement scmblable is celui qu'elle porte sur le précédent meidaillon. approche a grands pas yui font gonfler les piis de sa robe : de la main droile. elle lend vers le Ciente une couronne lemnistique. Paree qu'elle aurait inulilement surchargé la sciene. la palme n'apparail pas. Le troisieme médaillon (tip. 1:2. Ires frammentaire. represente la Victoire seule. élevant vers la gauche une couronne ${ }^{17}$. La parl ielle. destruction de l'objel ne permel pas de voir si la divinite port ail une palme.

Heres deux dernières représentations cst à retcuir la position très caractéristique de la main droite qui tend la couronme en arant, mais sans l'élerver au-dessus de l'épaule. la mourement. d'une ampleur retemur. correspond très précisément à colui que nous a deja montré la Vietoire de la saone.

Calle decourerte el l'hypothese que noms risquons at son sujet nous onl conduils a reprender l'examen du probleme des colomnes sur lescluelles les Victoires étaient dressées.

On sait que ces deux colonnes passent pour avoir été sciées par le milien et. vers le $\mathrm{xI}^{\mathrm{e}}$ sienele. transportens a Ainay où les quatre demi-füts soutiendraient aujourd'hui la roupole de la basilique saint-.llartin (fig. 13 ${ }^{18}$. Certes. il ne s'agit la que d'une tradition invérifiable. mais fondée sur des probabilités qui lui donnent une grande vraisemhlance et que confirment au surplus des travaux réecents. En 19:3). en effet. des observations faites lors de la destruetion du vieux pont de la ciuillotière ont révélé que les bloes antiques remployés dans cet ouvage. dont la ronstruction débuta en 1183. proviennent du sanc-

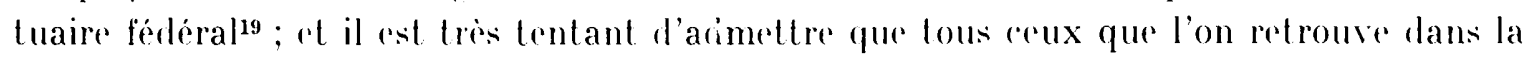
presqu'ile ot dont le remploi est antérieur a la fin du xire sièrle. c'est-i-dire a la construction de re pont. ont la mème origine : jusqu’a cedte époque. au plus tòt. la prérarité du

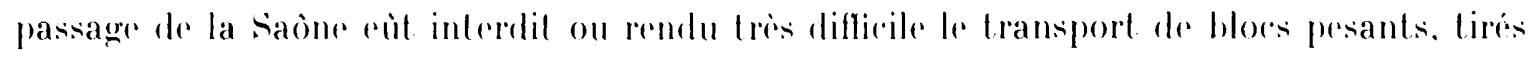
des édifiees de la ville haute. de Fourviere. (On peut done lenir pour tress vraisemblable que les colonnes d'Ainay ont eté apporteres, al partir du sanctuaire. par la vicille "charrièren de la rue Verciere, lacée des hords de l'amphithéatere jusque dans les parages de Bellecour.

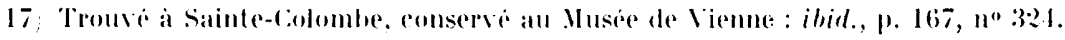

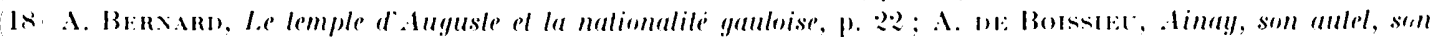

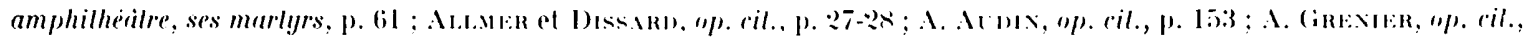
p. ว)غ.

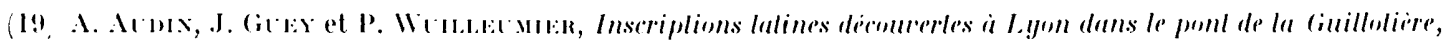
dans Rer. études anc.. I. 1 I, 1951, p. $297-3.16$, en particulier fig. p. 999. 


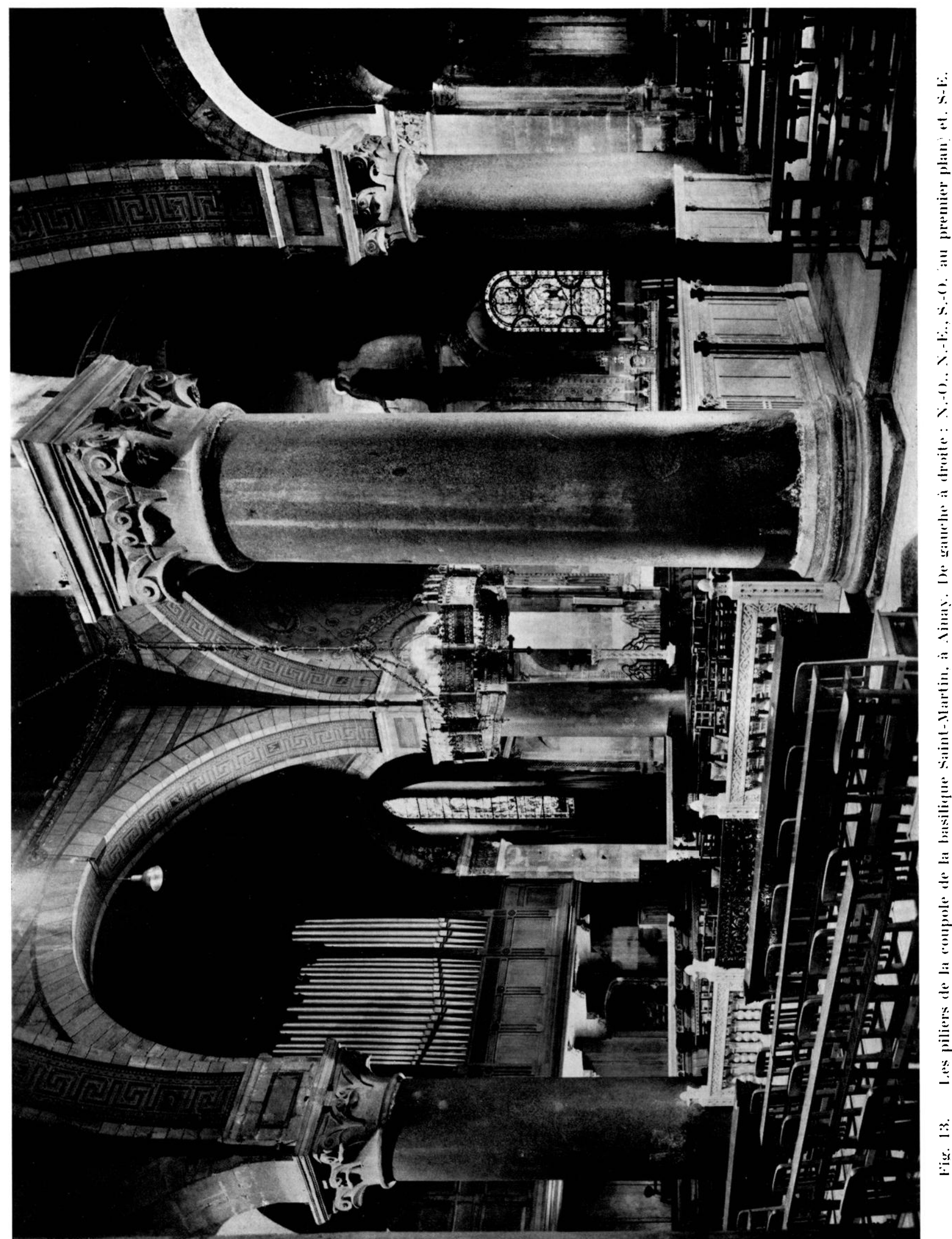


Leur caractère antique, en tout cas, ne fait aucun doute. Jes quatre puissants monolithes qui encadrent l'autel de la basilique sont d'un granil gris jaunàtre, rugueux et desquamé : une syénite importée de Ilaute-Égypte. A quelle autre époque qu'à l'époque gallo-romaine serait-on allé cherché si loin ce matériau ? En outre. cavel, listel el astragale sont là pour désigner. sans possibilité d'erreur, des colonnes antiques; le tailleur de pierre du Moyen Age eùt réservé toute mouluration aux bases el aux chapiteaux. Que. d'autre part. ces quatre piliers aient appartenu à deux colomness sciées ultérieurement par le milieu. il est aisé de s'en assurer : landis que le pilier surl-ouest comporte a son pied le cavet et le listel qui amorçaient la base moulurée. le pilier nord-est est toujours couronne par l'astragale limitant le chapiteau; détruits, ces éléments manquent sur les colonnes opposées. mais la trace en demeure visible; et l'on sail. depuis un quart de siecle ${ }^{20}$, que ces moiliés supérieures et inférieures de deux colonnes s'assemblent par croisement. le pilier nord-est se plaçant sur le pilier sud-ouest, le sud-est sur le nord-ouest. Jusqu'a présent. pourtant, aucune mensuration sérieuse des quatre piliers n'avait été entreprise qui permit de déterminer la hauteur des colonnes primitives et de se faire ainsi une idée plus précise de l’importance du monument auquel clles appartenaient. Ce délicat travail vient d'être effectué : nous en présentons les résultats.

Les fùts des colonnes antiques sont. on ne l'ignore pats. qalbés : ils s'évasent à part ir du sommet. jusqu'au tiers inférieur, auquel est résersé une forme cylindrique. Préjugeant de ce que les colonnes d'Linay répondaient i ce canon. nous pouvions espérer. en prenant comme base de calcul l'indice d'érasement, déterminer pour chacune d'elles la portion perdue au sciage dans le milieu du fùt. Mais la mensuration. ainsi pratiquée. se révéla décevante : en raison. d'abord. de l'altération du granit par endroit tres rongé ; i cause. ensuite. de l'irrégularité de l'évasement du füt : en plusieurs points, des renflements el des amincissements ont été comslalés. Au surplus, si, en un mème point le diamèt re du fût est généralement constant. parfois sonl apparues des diflérences assez sensibles selon qu'il élait levé sous une orientation ou sous une aut re. Linfin. la gìne résult ant. pour les deux piliers de l'bist. de la présence des stalles qui s'y adossent. n’a pas permis de les mesurer aver la plus grande précision. Il était done impossible de délerminer la hauteur des colonnes suivant la méthode entisagée.

Xous avons mème éprouré une singulière inquiélude lorsque aỵant vérifié que le pilier nord-est se plaçait sans difficulté sur le pilier sud-ouest, il apparul que la base du pilier sud-est, d'un diamitre de $1 \mathrm{~m} .076$, étail supérieure au sommet du pilier nord-ouest. d'un diametre de $1 \mathrm{~m}$. 067. sur lepuel elle venait reposer, différence que paraissait d'ailleurs accroilte la perte au sciage de la portion intermédiaire. Fallait-il en conclure que les quatre piliers d'anay provenaient de plus de deux colonnes anliques? que les colonnes d'où ils provenaient ne pouviaient donc itre identifiées aver celles de l'autel fédéral? I'n examen altentif du pilier sud-est. apportant la preuve du contraire. joue entierement en faveur de la tradition.

Au sommet, le diamelre de ce pilier est de 0 m. 985.). Immédial ement au-dessous. il se rél récil : $0 \mathrm{~m}$. 35) plus bas. il mesure $0 \mathrm{~m}$. 967 ; a part ir de la. il s'évase régulierement. pour alleindre $1 \mathrm{~m}$. 08:3 a 4 metres du sommet. Puis il s'amincil à nouveaul : a 4 m. 38. c'est-a-dire au pied du pilier. il n'est plus que de $1 \mathrm{~m}$. 076. Comme il n'y a pas apparence que cel amineissement anormal ail cessé brusquement en ce point, on ne s'élonne plus de constater que le diamètre supérieur de X.-O. est de $1 \mathrm{~m}$. 06z. Si bien que l'anomalie qui nous avait porté au doule oflre. finalement. la preuve que $\mathbf{S}$ - F. se plaçail bien sur X.-O. Preuve qui devient décisive à l'examen de ce dernier pilier, puisque ce n'esl qu'i 
$0 \mathrm{~m} .80$ du sommet qu'il retrouve le diamè tre de $1 \mathrm{~m} .076$ qui élail celui de la base de $\mathrm{S} .-\mathrm{L}$. Au-dessous. il s'évase à noureau pour atteindre. entre 2 mitres et $2 \mathrm{~m}$. 50). une porlion cylindrique de $1 \mathrm{~m}$. ().) de diametre. Nouvel évasement : a 4 metres du sommet. 1 m. 107. Puis. encore une fois. amincissement : a $4 \mathrm{~m}$. 20. c'est-i-dire juste au-dessus de la moulure de base. $1 \mathrm{~m} .105$.

A partir du taux d'amincissement observé à la base de s.-E. el au sommet de ..-O.. on preul cependant évaluer. approximativement. la perle au sciage entre les deux piliers ; correspondant i une différence de diamètre de 9 millimètres. elle n'est pas inférieure a $0 \mathrm{~m}$. 50. Pour retrouver la hauteur de la colonne, on additionnera done : $4 \mathrm{~m} .40$ (pilier $\mathrm{X} .-(0)+.0 \mathrm{~m}$. 50 (perte minimum au sciage) $4 \mathrm{~m} .38$ (pilier S.-E.) $+0 \mathrm{~m} .11$ (astragale de tite selon pilier X.-E.) $=9 \mathrm{~m}$. 39. Mais on se tiendra plus près de la réalité en ajoutant quelques centimètres à cette dimension ; et si l'on se souvient que 32 pieds romains font $9 \mathrm{~m}$. 47. ce dernier chiffre pourra ètre retenu comme théoriquement sat isfaisant ${ }^{21}$.

La seconde colonne se restitue en plaçant le pilier nord-est sur le pilier sud-ouest. Malheureusement. ces deux blocs. et surlout le second, présentent des desquamations qui risquent d'alterer les mesures. Sous l'astragale terminal. à $0 \mathrm{~m}$. 11 du sommet. le diamitre de S.-E. est de $0 \mathrm{~m}$. 971 . 11 se maintient tel sur une hauleur de quelque $1 \mathrm{~m}$. 75); et cette allure cylindrique est perceptible ¿ l'observateur. L'évasement ne débute qu'au-dessous, pour demeurer assez régulier jusqu'à 3 m. . of où le diamètre est de $1 \mathrm{~m}$. 006. A partir de là, il augmente brusquement ; ì $4 \mathrm{~mm}$. 30 du sommel, c'est-it-dire à la hase du pilier. il est de $1 \mathrm{~m}$. (227.

Le même évasement brusque se relrouve au sommet de. .-O.. dont le diamètre est de $1 \mathrm{~m} .047$. Il s'alténue ensuile. pour devenir peu sensible entre 2 et 3 mètres. oil il se tient aux alentours de $1 \mathrm{~m} .08$. Il reprend ensuite. atteint $1 \mathrm{~m} .108$ à 4 mètres, pour s'évaser brusquement à nouveau. Le diamètre levé à $4 \mathrm{~m}$. 15. au pied du pilier. est de $1 \mathrm{~m} / 11$ ). dimension que, en raison de reprises au ciment faites au pied du pilier. on peut ramener a $1 \mathrm{~m}$. 110. Le pied a perdu sa moulure terminale, brisée ; l'examen de $\mathrm{X}$.-O. révèle qu'elle devait mesurer $0 \mathrm{~m}$. 20 de haut.

Si les trois premiers piliers mesurent respectivement $4 \mathrm{~m}$. $30,4 \mathrm{~m}$. 38 et $4 \mathrm{~m}$. 40 de hauteur. S.-(). ne dépasse pas $4 \mathrm{~m}$. 15. Il semble que la portion correspondant à la moulure de pied de ce dernier ait élé aballue postérieurement au débitage des colonnes primitives : le pilier sud-ouest. mesurait alors, non $4 \mathrm{~m}$. 16, mais entre $4 \mathrm{~m}$. 36 et $4 \mathrm{~m}$. 40 . N'est-ce pas la ruplure accidentelle d'une partie de la moulure qui incila a la briser entierrement pour en retailler une neuve, intégrée il la base elle-mème, comme on peut le constater ?

Ia perte au sciage que trahil le déficit de 2 centimètres consiaté entre les diamètres mitoyens de X.-E. et de S.-O.. et qui correspond à la zone d'évasement rapide observé de part et d'autre, peul itre évaluée approximativement à $0 \mathrm{~m}$. 80 de haut. La hauteur lotale de la colonne résulterait donc de l'addition: $4 \mathrm{~m}$. 30 (pilier $\mathrm{X} . \mathrm{l}$. $)+0 \mathrm{~m} .80$ (perte approximative au sciage) $+4 \mathrm{~m} .16$ (pilier $:$ - (0.) + $0 \mathrm{~m} .20$ (moulure minimum du pied) $=9 \mathrm{~m}$. 45), chilfre qui comporte une part d'arbitraire dans le calcul de la perte au sciage. mais qui est roisin, en tout cas, de celui que nous avons obtenu pour la colonne précédente ${ }^{22}$.

Les deux colonnes deraient donc mesurer 3? pieds romains 23 . Égales en hauteur et de mème diamètre à la hase, elles présentaient, avec des irrégularités de profil, le mème aspect tronconique continu, assez inattendu dans un ouvrage de tradition classique : sans doute avaient-elles été taillées dans la carrière égyptienne, selon une technique ot des normes locales ${ }^{24}$.

(21) Sur cette hauteur, le diamitre du füt passe de $1 \mathrm{~m} .107$ (à $0 \mathrm{~m}$. 20 du bas à $0 \mathrm{~m} .967$ (à $0 \mathrm{~m}$. 35 du sommet , soit un amincissement de $0 \mathrm{~m} .14$, réduit à $0 \mathrm{~m} .12$ si l'on envisage les extrémités réelles du fùt.

Q? Le diametre passait de 0 m. 971 , sous l'astragale, a $1 \mathrm{~m}$. 110, au-dessus de la moulure du pied, soit un amincissement de 0 m. 139.

:33 Combre, remarquons-le, idealement pair, obtenu par donblement de tous les nombres à partir de l'unite.

:-4 X.-E. a reveli a son sommet un astragale haut de $0 \mathrm{~m}$. 07 , en saillie de $0 \mathrm{~m}$. 065 sur le fùt, soutenu par un 
Les deux fùts possédaient naturellement base ot chapiteau. Ies images monétaires leur attribuent une forme très simple, répondant à la nécessité de ne pas charger la décoration des colonnes et de réserver l'intérêt aux Victoires qu'elles portaient. Écartant done les ordres ionique et corinthien, nous nous en tiendrons à ce dorique simplifié qui sera adopté pour les colonnes décoratives impériales, Trajane et Antonine. La base de ce type. tore sur plinthe carrée. est égale en hauteur au rayon inférieur du fùt. pris comme unite modulaire, soit $0 \mathrm{~m}$. 5.) ; légèrement moins haut. le chapiteau associait une échine et un tailloir carré. Avere une approximation de peu d'amplitude. nous estimerons done la hauteur tolale des colonnes à $10 \mathrm{~m}$. 50).

Si. enfin, sur ces colomnes on place les Victoires, dont nous avons supposé qu'elles étaient deux fois plus grandes que nature, c'est-à-dire, en comptant les ailes qui dépassaient sensiblement la tète, hautes de $3 \mathrm{~m}$. jo) environ, nous obtenons au total, pour la colonne ef la Victoire, quatorze metres de hauteur ${ }^{25}$.

\section{A. Alime at P. Quoviam.}

listel de $0 \mathrm{~m}$. 025, en retrait de $0 \mathrm{~m}$. 0.4 sur le vif de l'astragale et porté par un cavet de $0 \mathrm{~m}$. 015 de haut et de $0 \mathrm{~m}$. 0 ? de creux : au total, $1 \mathrm{~m} .11$ de hauteur. -.- N.-O. a livre, a la hase, une plate-bande de $0 \mathrm{~m} .10$ de haut, en saillie de $0 \mathrm{~m} .07 .4$ sur le fùt, s amorgant par un cavet renversé de $0 \mathrm{~m}$. 10 de haut : au total, $0 \mathrm{~m}$. 20 de hauteur.

(25) Parmi les restes antiques decouverts dans les galeries des Fantasques, près de la couronne, figure egalement un objet de bronze dont nous avons donné plus haut la photographie (fig. 3j: l'angle d'un socle bas, haut de 0 m. 106. fixe sur une plaque debordante a lapuelle adhere un tenon informe, long encore de $0 \mathrm{~m}$. 07 a $0 \mathrm{~m}$. 08 et qui servail a sceller un monument de bronze sur une piece d'une nature differente. Signalons, toujours a titre d'hypothese, que cet objet pourrait provenir du socle sur le(puel se dressait une des Victoires et que le tenon fixait au chapiteaul dune des colonnes de laul el fidiral. 